\title{
Optimal Flux Magnitude Tracking with Application to Fault-tolerant Control of Wind Turbine Generators
}

\author{
Vinko Lešić, Student Member, IEEE, Mario Vašak, Member, IEEE, Nedjeljko Perić, Member, IEEE, \\ Gojko Joksimović, Member, IEEE, and Thomas M. Wolbank, Member, IEEE
}

\begin{abstract}
This paper concerns with wind turbine generator fault-tolerant control that avoids system shut-down and enables safe operation with less than nominal power production. We focus on generator stator isolation inter-turn fault that can be characterized before triggering the safety device and applied for any type of generator used in wind turbines. A low complexity model predictive tracking controller is proposed to achieve very accurate flux modulation that prevents the fault propagation while power delivery under fault is deteriorated as less as possible compared to healthy machine conditions. Presented fault-tolerant control strategy is developed taking into account its modular implementation and installation in available control systems of existing wind turbines to extend their life cycle and energy production. Simulation results for the case of a $700 \mathrm{~kW}$ wind turbine are presented.
\end{abstract}

\section{INTRODUCTION}

Renewable energy sources made a great political and economic impact in the last 20 years and encouraged an energy revolution that affected the whole world. After a huge breakthrough and an average growth rate of $26 \%$ in last 5 years, wind energy today is a well-established technology with total world installed capacity of approximately $238 \mathrm{GW}$ at the end of 2011 [1]. However, market competence of renewables and wind energy is an everlasting debate. Different branches of science put a lot of effort into making renewables a technology we can fully rely on.

Remote locations are best suitable for wind turbines operation because of low-turbulent and strong winds. This introduces difficult and expensive maintenance procedures and rises availability concerns. Several fault-tolerant control (FTC) algorithms have been introduced in [2] and they mostly propose different kinds of redundancies for sensors and electronic components. Focus here is on generator electromechanical faults, which are besides gearbox and power converters faults the most common in wind turbine systems [3]. Following the rising share of direct-drive wind turbines $(18 \%$ of the market in 2010 [1]) and thereby the usage of more complex and more fault-prone generators, the focus on generator faults in wind turbines is expected to further increase.

In our recent paper [4] we proposed an FTC, built on fault detection and characterization procedure proposed in [5],

Vinko Lešić, Mario Vašak and Nedjeljko Perić are with the University of Zagreb, Faculty of Electrical Engineering and Computing, Advanced Control Team group, Zagreb, Croatia (e-mail: vinko.lesic@fer.hr)

Gojko Joksimović is with the University of Montenegro, Faculty of Electrical Engineering, Podgorica, Montenegro

Thomas M. Wolbank is with the Vienna University of Technology, Faculty of Electrical Engineering and Information Technology, Vienna, Austria which enables generator operation in the case of isolation degradation and inter-turn short circuit in a stator winding phase. Stator winding isolation faults cause about $35 \%$ of machine faults [6][7] and are common for both asynchronous and synchronous machines used in wind turbines (doubly-fed induction generator, squirrel-cage induction generator, synchronous generator with wound rotor, synchronous generator with permanent magnets).

The FTC avoids wind turbine shut-down and enables subnominal power production until next scheduled overhaul. The algorithm is based on proper extension of widely adopted control strategies used in wind turbines, mainly on torque control loop with field-oriented control (FOC). An outer control loop is designed to utilize FOC and achieve proper machine flux modulation that stops further fault development while power delivery under fault is deteriorated as less as possible compared to healthy machine conditions. The whole procedure needs to be very accurate and performed with the high frequency of voltage supplied to the machine stator.

The flux modulation is periodical whereas the machine stator flux is strengthened and weakened in a way that its derivative never exceeds the limit imposed by the fault characterization procedure. The problem of implementing the flux modulation can be therefore observed as a reference tracking problem. The reference can be calculated in advance, which gives a fine opportunity for applying a modelpredictive control (MPC) approach.

The main issue is that flux modulation has to be executed at each discrete time step and sample time used for FOC is required to be in hundred of microseconds scale. This imposes a severe limit to the MPC algorithm complexity and computational effort possibilities.

Focus of this paper is to develop a very fast model predictive controller that ensures the stator flux reference tracking and enables proper operation of the outer FTC loop. Described algorithm is derived for the case of squirrel-cage induction generator (SCIG) but whole FTC can be similarly applied for any type of a generator used in wind turbines.

This paper is organized as follows. The mathematical model of an SCIG and basic rotor FOC are presented in Section II. A fault-tolerant approach and control algorithm that enables wind turbine operation under stator isolation fault is described in Section III. Section IV focuses on the development of MPC and stator flux reference tracking. Section V provides simulation results for the case of $700 \mathrm{~kW}$ wind turbine. Conclusions are given in Section VI. 


\section{Mathematical Model OF AN INDUCTION MACHine}

A very common mathematical model of an AC squirrelcage induction machine represented in the two-phase $(d, q)$ rotating coordinate system and with rotor flux linkage aligned with the $d$-axis (rotor flux vector $\bar{\psi}_{r}$ is set to $\psi_{r d}$ ) is described:

$$
\begin{aligned}
& u_{s d}+\Delta u_{s d}=k_{s} i_{s d}+L_{l} \frac{d i_{s d}}{d t}, \\
& u_{s q}+\Delta u_{s q}=R_{s} i_{s q}+L_{l} \frac{d i_{s q}}{d t},
\end{aligned}
$$

with $L_{l}=\left(L_{s}-\frac{L_{m}^{2}}{L_{r}}\right)$ and $k_{s}=\left(R_{s}-\frac{L_{l}}{T_{r}}+\frac{L_{s}}{T_{r}}\right)$. Variables $u_{s d, q}$ are stator phase voltages in $(d, q)$ coordinate system, $i_{s d, q}$ are stator phase currents, $L_{s}, L_{r}$ and $L_{m}$ are stator, rotor and mutual inductances, respectively. Parameters $R_{s}$ and $R_{r}$ are stator and rotor resistances and $T_{r}=\frac{L_{r}}{R_{r}}$ is the rotor time constant. Voltages $\Delta u_{s d}$ and $\Delta u_{s q}$ are called decoupling or correction voltages:

$$
\begin{aligned}
\Delta u_{s d} & =\frac{1}{T_{r}} \frac{L_{m}^{2}}{L_{r}} i_{m r}+\omega_{e} L_{l} i_{s q}, \\
\Delta u_{s q} & =-\omega_{e} \frac{L_{m}^{2}}{L_{r}} i_{m r}-\omega_{e} L_{l} i_{s d},
\end{aligned}
$$

which ensure that the voltage value in one axis is not affected by the voltage in other. The rotor flux is created by the magnetizing current $i_{m r}$ and $\omega_{e}$ is the frequency of voltage supplied to the stator. A rotor field-oriented control (RFOC) equations from which the AC machine model (1)-(4) is derived are:

$$
\begin{aligned}
i_{m r} & =\frac{\psi_{r d}}{L_{m}} \\
i_{s d} & =i_{m r}+T_{r} \frac{d i_{m r}}{d t}, \\
\omega_{s l} & =\omega_{e}-p \omega_{g}, \\
T_{g} & =\frac{3}{2} p \frac{L_{m}^{2}}{L_{r}} i_{m r} i_{s q}=k_{m} i_{m r} i_{s q},
\end{aligned}
$$

where $\omega_{s l}=\frac{i_{s q}}{T_{r} i_{m r}}$ is the slip speed, $\omega_{g}$ is the mechanical speed, $p$ denotes the number of pole pairs and $T_{g}$ is the electromagnetic torque. The torque is controlled only by $q$ stator current component because magnetizing current vector $i_{m r}$ is dependent on the time lag $T_{r}$ and is therefore kept constant in the sub-nominal speed operating region.

Machine model given by (1) and (2) is suitable for proportional-integral (PI) controller design. If integral time constants $T_{I d}=L_{l} / k_{s}$ for $d$-current, $T_{I q}=L_{l} / R_{s}$ for $q$ current and gain $K_{r}$ are chosen, closed loop dynamics can be represented as a first-order lag system with transfer function:

$$
\frac{i_{s d}(s)}{i_{s d}^{*}(s)}=\frac{i_{s q}(s)}{i_{s q}^{*}(s)}=\frac{1}{1+\tau s},
$$

where $\tau$ is a time constant defined with $\tau=\frac{L_{l}}{K_{r}}$. For more information about machine modelling and FOC please refer to [8].

\section{FAULT-TOLERANT CONTROL}

Some of the most common causes of stator isolation faults are moisture in the isolation, winding overheating, or vibrations. Modern voltage-source inverters also introduce additional voltage stress on the inter-turn isolation caused by the steep-fronted voltage surge [6].

If there is a short circuit between turns of the same phase the time elapsed between incipient fault and triggered safety device is about several minutes or even much longer, depending on the stator winding method [7]. This may give enough time for fault detection and adequate autonomous reaction provided by control system to suppress the fault from further spreading on other wind turbine components.

The fault is manifested as a shorted turn in the phase winding with very low resistance. It results in high currents that flow through that turn and cause machine torque reduction and local overheating. The goal of considered FTC is to keep the current in the shorted turn below its rated value and stop the fault from spreading on other components. This causes the wind turbine to operate at below-rated power but protects the generator until scheduled repair. This is very important for remotely-located wind turbines since the shorted turns induce further fault spreading up to the point where the whole generator needs to be replaced.

The current flow is caused by induced voltage due to variable magnetic flux and can be therefore restricted by manipulation of the flux. In the three-phase coordinate system $(a, b, c)$ stator voltage equation is defined with:

$$
u_{s x}=i_{s x} R_{s}+\frac{d \psi_{s x}}{d t} \approx \frac{d \psi_{s x}}{d t},
$$

where $x$ denotes one of the phases. The goal for suppressing the fault is formed as a restricted value of flux derivative:

$$
\left|\frac{d \psi_{s x}}{d t}\right| \leq K
$$

The value $K$ is determined based on fault identification procedure through machine fault monitoring and characterization techniques [5]. Following from (10) this restricts the induced voltage in the faulted turns and consequently also the circulating currents responsible for local overheating. Generally, the stator flux is considered sine-wave (in the fundamental-wave approaches, such as FOC) with amplitude $\left|\psi_{s}\right|$, angular frequency $\omega_{e}$ and phase offset $\varphi_{x}$. The flux in phases $x=a, b, c$ is represented with:

$$
\psi_{s x}(t)=\left|\psi_{s}\right|(t) \sin \left(\omega_{e} t+\varphi_{x}\right) .
$$

Flux amplitude envelope denoted with $\left|\psi_{s}\right|(t)$ in (12) is timevariable and must be taken into account when calculating the flux derivative for fault suppression:

$$
\frac{d \psi_{s x}(t)}{d t}=\frac{d\left|\psi_{s}\right|(t)}{d t} \sin \left(\omega_{e} t\right)+\left|\psi_{s}\right|(t) \omega_{e} \cos \left(\omega_{e} t\right) .
$$

Considering the design of fault-tolerant control strategy, one approach is to use constant but weakened flux $\psi_{s}$ such that $\left|\psi_{s}\right| \omega_{e}$ is kept below value of $K$. Wind turbine pitch control is used then to lower the aerodynamic torque in order to impose the torque balance. The weakened flux is shown 
in Fig. 1 as Faulty $A$ waveform. The approach lowers the generator power production unnecessarily. In the sequel we present a method for significantly improving the wind turbine power production while suppressing the fault at the same time.

From (11) it follows that in order to stop the fault from spreading, the stator flux waveform must never be allowed to exceed a restriction shaped as a triangular waveform $|K t|$ shown in Fig. 1 (dash-dot). The restriction also represents the flux waveform that enables maximum power production in the faulty machine state. Therefore, our goal is to utilize the existing control strategy and FOC to achieve the triangular waveform:

$$
\begin{aligned}
& \psi_{s a}(t)=K t, \quad t \in\left[-\frac{\pi}{2 \omega_{e}}, \frac{\pi}{2 \omega_{e}}\right]+\frac{2 k \pi}{\omega_{e}} \\
& \psi_{s a}(t)=-K t+K \pi, \quad t \in\left[\frac{\pi}{2 \omega_{e}}, \frac{3 \pi}{2 \omega_{e}}\right]+\frac{2 k \pi}{\omega_{e}}
\end{aligned}
$$

An appropriate flux amplitude envelope is chosen such that $\left|\frac{d \psi_{s x}}{d t}\right|=K$ (for phase $a$ with $\varphi_{a}=0$ ):

$$
\left|\psi_{s}\right|(t)=\frac{\frac{K}{\omega_{e}} \omega_{e} t}{\sin \left(\omega_{e} t\right)},
$$

with minimum absolute value at angles $\omega_{e} t=0, \pi, \ldots$, and maximum at $\omega_{e} t=\pi / 2,3 \pi / 2 \ldots$ :

$$
\begin{aligned}
\left|\psi_{s}\right|(0) & =\frac{K}{\omega_{e}}, \\
\left|\psi_{s}\right|\left(\frac{\pi}{2}\right) & =\frac{K}{\omega_{e}} \frac{\pi}{2} .
\end{aligned}
$$

Transformed to the $(d, q)$ and RFOC domain, stator flux linkage is defined with $\bar{\psi}_{s}=\psi_{s d}+j \psi_{s q}$ where:

$$
\begin{aligned}
& \psi_{s d}=L_{l} i_{s d}+\frac{L_{m}}{L_{r}} \psi_{r d}, \\
& \psi_{s q}=L_{l} i_{s q},
\end{aligned}
$$

and relation $\left|\psi_{s}\right|=\sqrt{\psi_{s d}^{2}+\psi_{s q}^{2}}$ holds.

Changing the $\psi_{r d}$ is very slow due to large rotor time-lag $T_{r}$, so we choose to manipulate the flux $\psi_{s x}$ only through machine fast dynamics (9). Proper values of current components $i_{s d}$ and $i_{s q}$ are chosen to achieve the triangular form in Fig. 1 while taking into account: (i) $\left|i_{s}\right|$ must not exceed predefined nominal value $i_{s n}$, (ii) desired machine torque and corresponding $i_{s q}$, (iii) the maximum flux restriction (rated value) due to saturation. The value of $\psi_{r d}$ is considered constant during the manipulation of stator flux. In practice

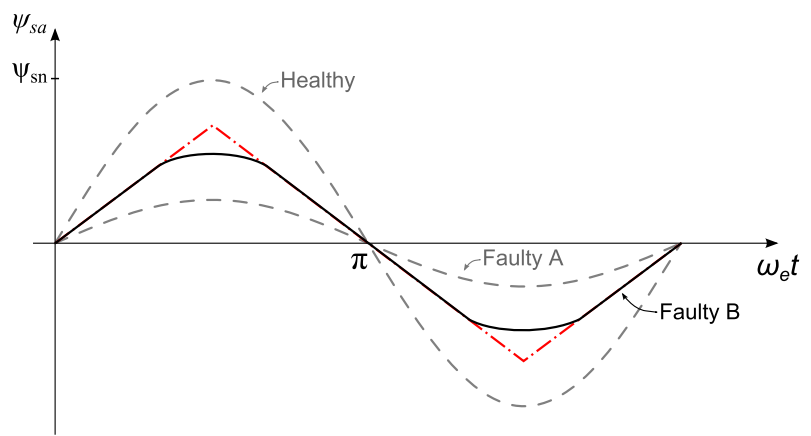

Fig. 1. Stator flux waveforms for healthy and faulty machine. it is influenced by changing the $i_{s d}$ current and it slowly fluctuates around mean value of the chosen $\psi_{r d}$.

If parameter $K$ from the fault condition (11) is too large or chosen $\psi_{r d}$ value limits the freedom for desired fast dynamics, desired amplitude scope shown in Fig. 2 with peak values from (16) is not achievable. Therefore the minimum value is set to $\psi_{s_{-} \text {min }}=\frac{K}{\omega_{e}}$ and when the amplitude envelope $\left|\psi_{s}\right|(t)$ reaches value of $\psi_{s \_ \text {max }}$ (at time instant $t_{m}$ ) it is fixed to that value. The objective (11) is still achieved with slightly reduced maximum possible power production and obeyed machine constraints. Stator flux takes the form presented as Faulty $B$ in Fig. 1. The amplitude envelope is shown in Fig. 2 with dash-dot line representing the case when triangular waveform is achievable and full line for the case of Faulty $B$ waveform. Note that amplitude envelope frequency is twice the frequency of desired Faulty $B$ waveform. The time instant $t_{m}$ can be obtained from condition:

$$
K t_{m}=\psi_{s_{-} \max } \sin \left(\omega_{e} t_{m}\right) .
$$

As the $q$-current is responsible for maintaining the constant torque, the $d$-current dynamics determines, according to (17), the dynamics of $\left|\psi_{s}\right|(t)$. Fastest achievable transient of $i_{s d}$ is determined with inverter limitation. Considering the worst case scenario and a typical value of $U_{d c}$ in wind turbines, even the maximum stator flux derivative at $t=\frac{\pi}{2 \omega_{e}}$ is achievable and this issue is not explicitly treated in the derived FTC.

Desired generator torque reference $T_{g}^{*}$ dictated by the wind turbine torque control loop [9] determines adequate value of $\omega_{e}$ and $i_{m r}$ (or $\psi_{r d}$ ) used to achieve correct $\psi_{s \_ \text {min }}$ and $\psi_{s_{-} \max }$. Current references $i_{s d}^{*}$ and $i_{s q}^{*}$ are then calculated to obtain desired stator flux wave. The algorithm is given in the Algorithm 1.

Step 6 is executed at each discrete time step, while steps 1-5 are executed once in every stator flux modulation period (Fig. 2) for less computational effort. Steps 1-5 require solving of simple algebraic equations i.e. quadratic and square root equations but can be simplified even more using look-up tables. Initialization of the algorithm is executed only once when a fault is diagnosed and can also be obtained from look-up table $\omega_{g 1}(K)$.

Using the described method, an exemplary graph of available speed-torque points under machine fault is shown in Fig. 3. Normal operation of the healthy generator is bounded

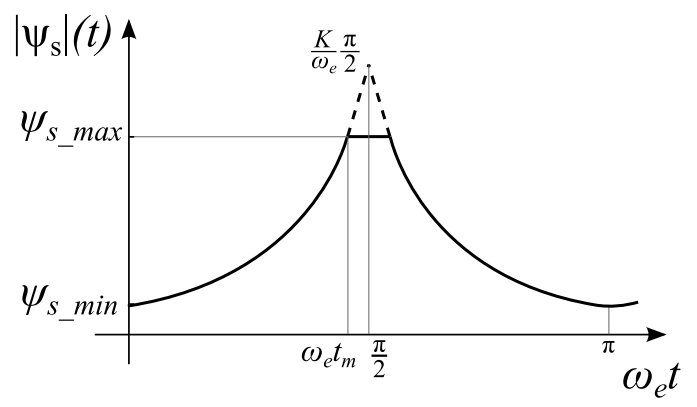

Fig. 2. Flux amplitude from (15) for achieving $\left|\frac{d \psi_{s x}}{d t}\right| \leq K$. 


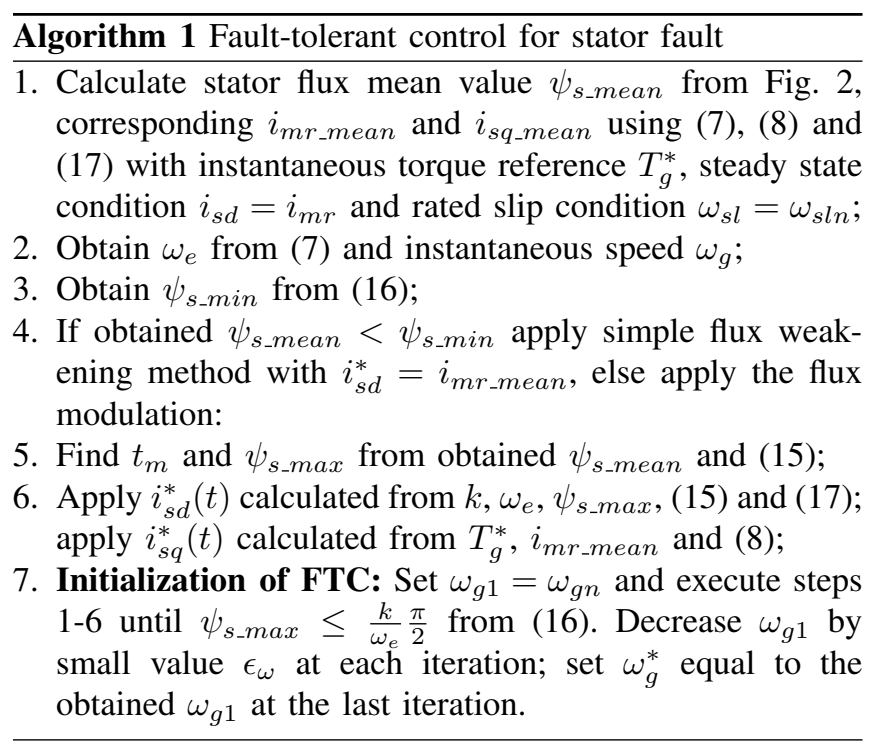

with rated machine torque $T_{g n}$ and rated speed $\omega_{g n}$ and pitch control is responsible to keep the operating point between boundaries. The curve denoted with Optimum power represents optimal operating points of wind turbine at which the power factor coefficient $C_{p}$ and power production is at maximum value. Optimum power production is entrusted to torque controller [4][9].

For the case of diagnosed fault, dashed area denotes all available generator torque values that can be achieved for certain generator speed. Generator torque denoted with $T_{f}$ is the largest available torque that can be achieved under fault condition. Below speed $\omega_{g}^{\prime}$ generator is operating in the safety region and no fault-tolerant control is needed. It follows that up to the speed $\omega_{g 1}$ it is possible to control the wind turbine in the faulty case without sacrificing power production. However, from that speed onwards it is necessary to use blades pitching in order to limit the aerodynamic torque and to keep the power production below optimal in order to suppress the fault from spreading. The speed control loop is modified such that instead of reference $\omega_{g n}$ the reference $\omega_{g 1}$ is selected. Interventions in classical variable

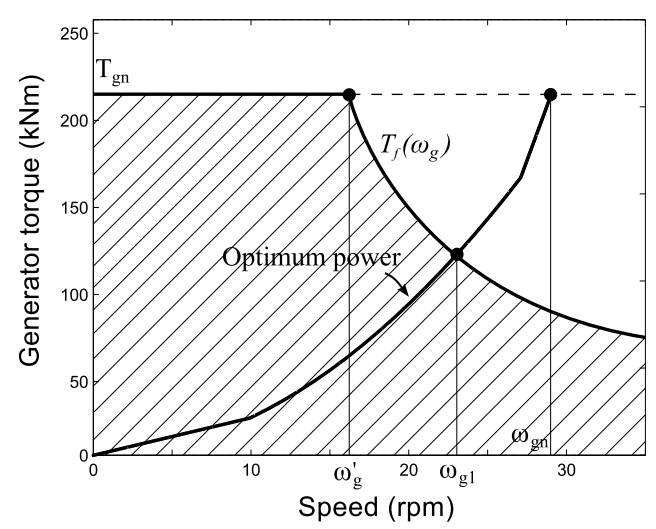

Fig. 3. Available torque-speed generator operating points under fault condition (shaded area)

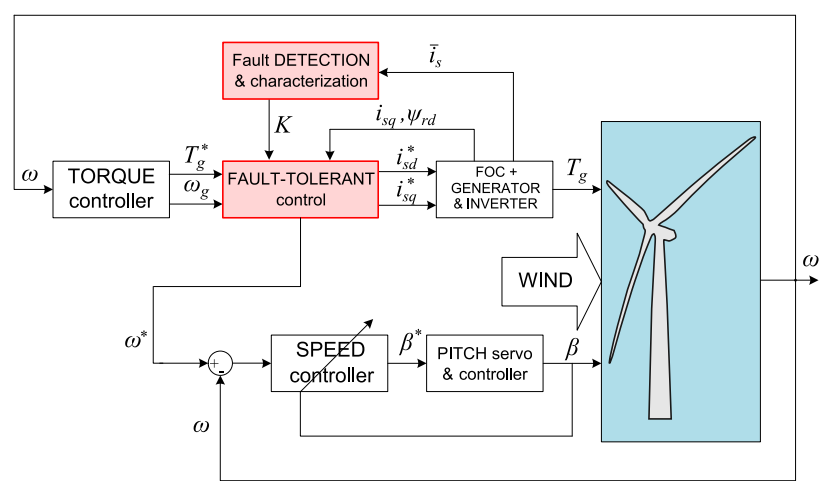

Fig. 4. Control system of wind turbine with fault-tolerant control strategy.

speed variable pitch wind turbine control that ensure faulttolerant operation are given in Fig. 4 (red blocks).

\section{MODEL PREDICTIVE CONTROL}

In [4] we relied on inner FOC current control loops for flux modulation, which have introduced overshoot of the flux derivative constraint $K$. To overcome the problem we used lower value of $K$ constraint as a redundancy, which also resulted in unnecessary loss of the power production.

Focus here is to achieve higher power production with model predictive control based on the presented induction machine model. The prediction of states and reference values is therefore used in computation of control law. For that purpose we discuss optimal control for the flux modulation reference tracking. The algorithm has to be implemented on-line and applied at every time instant while reference tracking has to be performed very accurately to fulfill the task of the outer FTC loop to restrain the fault propagation. From the controller-design aspect, the first requirement is the crucial one and algorithm has to be of low complexity to fit in the FOC sample time scale, which is usually about hundred of microseconds. Since the modulation is performed periodically, with maximum of one period ahead of known reference, using a conventional linear quadratic regulator (LQR) with infinite horizon problem is not an option.

We use therefore an unconstrained quadratic optimal control approach with finite prediction horizon [10]. Respecting of constraints is performed by the slow FTC loop, which is executed once per flux modulation period. The task of tracking controller is to calculate the correct $d$-current reference and pass it forward to the FOC current control loop in such way that desired stator flux reference is accomplished. Both $d$-current and stator flux dynamics (6) and (9) have to be included in the design process:

$$
\begin{aligned}
\frac{i_{s d}(s)}{i_{s d}^{*}(s)} & =\frac{1}{1+\tau s}, \\
\frac{\psi_{r d}(s)}{i_{s d}(s)} & =\frac{L_{m}}{1+T_{r} s} .
\end{aligned}
$$

The problem is therefore formed as control of a discrete two-state linear system:

$$
\begin{aligned}
x_{k+1} & =\mathbf{A} x_{k}+\mathbf{B} u_{k}, \\
y_{k} & =\mathbf{C} x_{k},
\end{aligned}
$$


where states and inputs are:

$$
x=\left[\begin{array}{c}
i_{s d} \\
\psi_{r d}
\end{array}\right], \quad u=i_{s d}^{*} .
$$

The $\psi_{s}$ from (17) comprises $i_{s d}$ and $\psi_{r d}$ in the squareroot quadratic sum and using it in the quadratic criteria of the cost function would be too complex. Since $\psi_{s d}$ holds the majority of the stator flux $\psi_{s}$ (more than 99\% in our case) and $\psi_{s q}$ is practically constant we choose not to consider $\psi_{s q}\left(i_{s q}\right)$ dynamics. This way the forming of cost function and minimization criteria is significantly simplified. Therefore, with $y_{k}=\psi_{s d, k}$, state, input and output matrices of the system are:

$$
\begin{gathered}
\mathbf{A}=\left[\begin{array}{cc}
1-\frac{T_{s}}{\tau} & 0 \\
\frac{T_{s}}{T_{r}} & 1-\frac{T_{s}}{\tau}
\end{array}\right], \quad \mathbf{B}=\left[\begin{array}{c}
\frac{T_{s}}{\tau} \\
0
\end{array}\right], \\
\mathbf{C}=\left[\begin{array}{cc}
L_{l} & \frac{L_{m}}{L_{r}}
\end{array}\right],
\end{gathered}
$$

where $T_{s}$ is the sampling time.

Cost function for tracking problem is chosen:

$$
J=\sum_{k=0}^{N} q_{k}\left(y_{k}-r_{k}\right)^{2},
$$

where $N, r_{k}, q_{k}$ are prediction horizon, reference prediction and tracking error weighting factor, respectively. The penalization of the control signal $u$ is left out because of very dynamic flux modulation trajectory changing.

Generally, states in time-step $k$ can be described with:

$$
x_{k}=\mathbf{A}^{k} x_{0}+\sum_{i=0}^{k-1} \mathbf{A}^{i} \mathbf{B} u_{i},
$$

and represented in the matrix form as:

$$
\begin{aligned}
\mathcal{X} & =\mathbf{A}^{*} x_{0}+\mathbf{B}^{*} \mathcal{U} \\
\mathcal{Y} & =\mathbf{C}^{*} \mathcal{X}
\end{aligned}
$$

where $\mathcal{X}, \mathcal{U}, \mathcal{Y}, \mathbf{A}^{*}, \mathbf{B}^{*}, \mathbf{C}^{*}$ are model matrices on the prediction horizon $N$.

The quadratic cost function is now:

$$
J=\left(\mathbf{C}^{*} \mathcal{X}-\mathbf{R}\right)^{\top} \mathbf{Q}\left(\mathbf{C}^{*} \mathcal{X}-\mathbf{R}\right),
$$

where $\mathbf{R}=\left[\begin{array}{llll}r_{0} & r_{1} & \ldots & r_{N}\end{array}\right]^{\top}$ is a vector of future reference values. By putting model matrices, the cost function obtains the final form:

$$
J=\mathcal{U}^{\top} \mathcal{H} \mathcal{U}+f \mathcal{U}+g
$$

where

$$
\begin{aligned}
\mathcal{H} & =\mathbf{B}^{* \top} \mathbf{C}^{* \top} \mathbf{Q} \mathbf{C}^{*} \mathbf{B}^{*}, \\
f & =2 x_{0}^{\top} \mathbf{A}^{* \top} \mathbf{C}^{* \top} \mathbf{Q} \mathbf{C}^{*} \mathbf{B}^{*}-2 \mathbf{R}^{\top} \mathbf{Q} \mathbf{C}^{*} \mathbf{B}^{*}, \\
g & =x_{0}^{\top} \mathbf{A}^{* \top} \mathbf{C}^{* \top} \mathbf{Q} \mathbf{C}^{*} \mathbf{A}^{*} x_{0}-2 \mathbf{R}^{\top} \mathbf{Q} \mathbf{C}^{*} \mathbf{A}^{*} x_{0}+\mathbf{R}^{\top} \mathbf{Q R} .
\end{aligned}
$$

The solution that minimizes $J[10][11]$ is given by:

$$
\mathcal{U}^{*}=-\frac{1}{2} \mathcal{H}^{-1} f^{\top} .
$$

It may be observed that $\mathcal{H}^{-1}$ is a constant matrix and can be calculated off-line. This significantly decreases the algorithm computational effort and improves the execution speed. The reference values $\mathbf{R}$ are predicted using (15), (17) and (18) to achieve the waveform from Fig. 2.

From (7) it follows that $\omega_{e}$ is dependent on $i_{s d}$ and $\psi_{r d}$, which is not considered in predictive control. Instead, the weighting matrix $Q$ is chosen to enhance the influence of closer prediction steps and attenuate the farther. Elements of diagonal matrix $Q$ are obtained from:

$$
q_{i, i}=1.1^{-i}, \quad i=0, \ldots, N
$$

Estimation of FOC variables [8] is exploited to form the initial state vector $x_{0}=\left[\begin{array}{ll}i_{s d 0} & \psi_{r d 0}\end{array}\right]^{\top}$ for each time step. Control law is applied based on receding horizon control approach whereas $u_{0}$ is segregated from $\mathcal{U}^{*}$ and passed to inner current FOC loops. The MPC controller is used to calculate the $i_{s d}^{*}$ in Step 6 of Algorithm 1 .

\section{Simulation Results}

This section provides simulation results for a $700 \mathrm{~kW}$ MATLAB/Simulink variable-speed variable-pitch wind turbine model with a two-pole $5.5 \mathrm{~kW}$ SCIG scaled to match the torque and power of $700 \mathrm{~kW}$ machine.

Generator parameters are: $p=1, U_{n}=186.67 \mathrm{~V}, I_{n}=$ $14.07 \mathrm{~A}, f_{n}=50 \mathrm{~Hz}, L_{s}=L_{r}=0.112 \mathrm{H}, L_{m}=0.11$ $\mathrm{H}, R_{s}=0.3304 \Omega, R_{r}=0.2334 \Omega$ and PI controller gain is $K_{r}=6$. Stator flux rated value is $\psi_{s n}=0.625 \mathrm{~Wb}$ and sample time is chosen $T_{s}=2 \cdot 10^{-4} \mathrm{~s}$. Moment of inertia of wind turbine and generator, reduced to the generator side is $J=72.11 \mathrm{kgm}^{2}$.

The prediction horizon of $N=10$ has proven adequate for presented control problem. For FOC variables estimation we use an unscented Kalman filter proposed in [12], and for this particular purpose applied in [13]. In the sequel simulation results with diagnosed fault and $\left|\frac{d \psi_{s x}}{d t}\right|=K=100 \mathrm{~Wb} / \mathrm{s}$ are presented. The $i_{s q}$ current is used only to compensate slow $i_{m r}$ fluctuations in order to maintain the desired torque and stator flux is manipulated only by $i_{s d}$ as presented in Fig. 5. The figure also shows influence of predicted machine dynamics used in $i_{s d}$ reference calculations.

Figure 6 shows very accurate reference tracking of stator flux amplitude envelope from (15) with the proposed model predictive controller. Corresponding stator flux waveforms in phases $a, b, c$ are shown in Fig. 8. Following from figures,

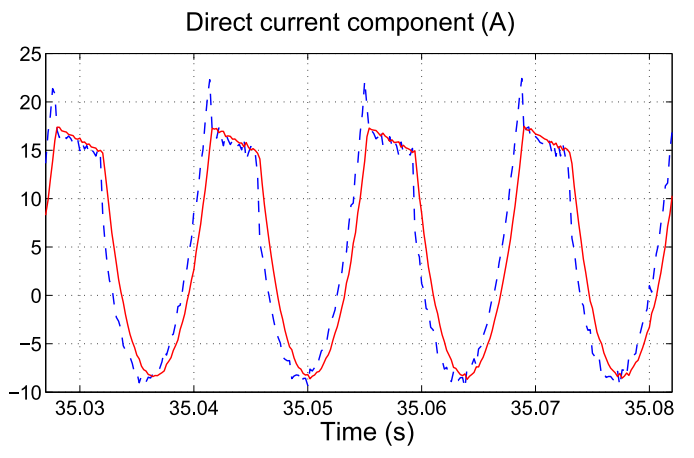

Fig. 5. Direct current component. Dashed is the reference value. 


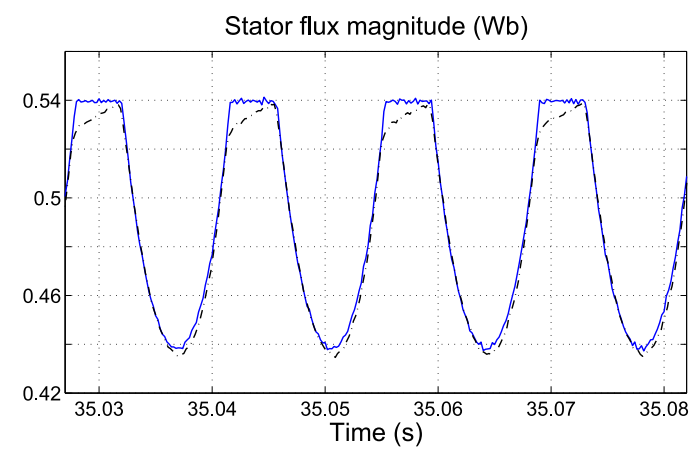

Fig. 6. Stator flux magnitude $\left|\psi_{s}\right|(t)$. Dash-dot line is for the case without MPC.

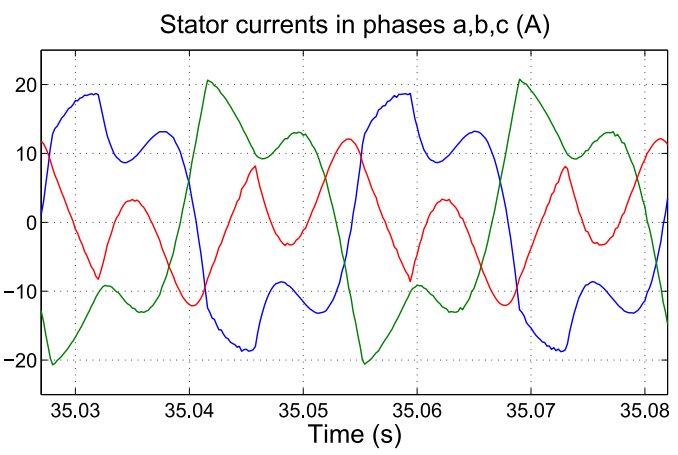

Fig. 7. Stator phase currents.

the required restriction formed as triangular waveform is satisfied in the targeted phase (denoted as faulty phase in figures 8 and 9) and the flux derivation never exceeds the restriction $K$ (Fig. 9). Comparison with the flux modulation achieved without described predictive controller is also given in figures. Phase currents calculated to achieve desired flux waveforms are shown in Fig. 7. Currents follow a sinusoidal shape with deviations at peak values.

\section{Conclusions}

An extension of the conventional wind turbine control structure is proposed to prevent the generator stator isolation fault propagation and enable faulty wind turbine operation with reduced power. The method is presented on a squirrelcage generator with diagnosed short circuit between turns of the same phase. A low complexity, explicit model predictive controller has been proposed for very accurate flux modulation tracking. The power delivery under fault is deteriorated as less as possible compared to healthy machine conditions.

\section{ACKNOWLEDGEMENT}

The research leading to these results has received funding from the European Communitys Seventh Framework Programme under grant agreement no 285939 (ACROSS).

\section{REFERENCES}

[1] Renewable Energy Policy Network for the 21st Century (REN21), "Renewables 2012", Global status report, 2012.

[2] S. Pourmohammad, A. Fekih, "Fault-Tolerant Control of Wind Turbine Systems - A Review",Proc. of the 2011. IEEE Green Technologies Conference (IEEE-Green), April 2011.

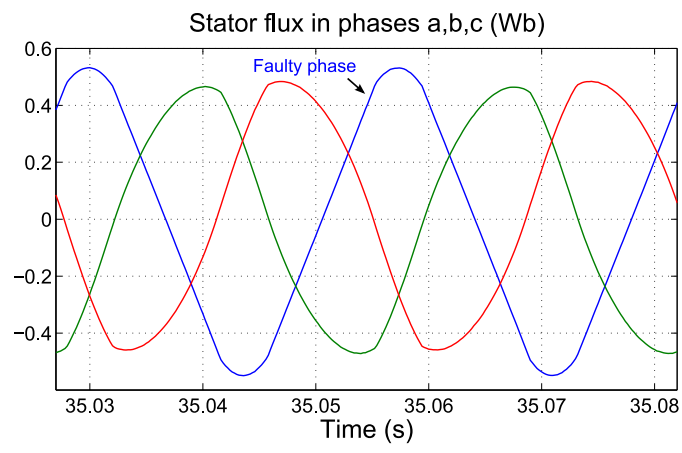

Fig. 8. Stator flux in phases $a, b, c$.

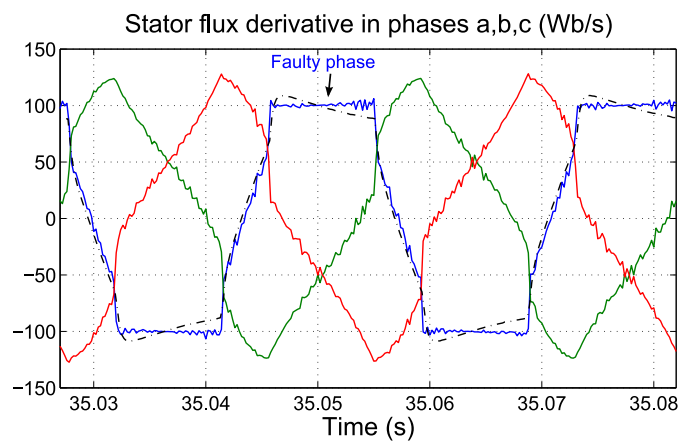

Fig. 9. Stator flux derivative in phases $a, b, c$. Dash-dot line is for the case without MPC.

[3] Z. Daneshi-Far, G. A. Capolino, H. Henao, "Review of Failures and Condition Monitoring in Wind Turbine Generators", XIX International Conference on Electrical Machines - ICEM 2010, September 2010.

[4] V. Lešić, M. Vašak, N. Perić, G. Joksimović and T. M. Wolbank, "Faulttolerant Control of a Wind Turbine with a Squirrel-cage Induction Generator and Stator Inter-turn Faults", The 12th International Workshop on Advanced Motion Control, AMC, March 2012.

[5] G. Stojičić, G. Joksimović, M. Vašak, N. Perić and T. M. Wolbank, ’Increasing Sensitivity of Stator Winding Short Circuit Fault Indicator in Inverter Fed Induction Machines", 15th International Power Electronics and Motion Control Conference, EPE-PEMC ECCE Europe 2012, pp. DS2a.10-1-7, Sept. 2012.

[6] A. H. Bonnet and C. Yung, "Increased efficiency versus increased reliability", IEEE Industry Applications Magazine, vol.14, no.1, pp. 2936, 2008.

[7] O. Thorsen and M. Dalva, "A survey of faults on induction motors in offshore oil industry, petrochemical industry, gas terminals, and oil refineries", IEEE Transactions on Industry Applications, vol.31, pp. 1186-1196, Sept./Oct. 1995.

[8] M. P. Kazmierkowski, F. Blaabjerg and R. Krishnan, Control in Power Electronics - Selected Problems, San Diego, California: Academic Press, An imprint of Elsevier Science, ISBN 0-12-402772-5, 2002.

[9] T. Burton, D. Sharpe, N. Jenkins and E. Bossanyi Wind Energy Handbook, England: John Wiley \& Sons, ISBN 0-471-48997-2, 2001.

[10] F. Borrelli, Constrained Optimal Control of Linear and Hybrid Systems, volume 290 of Lecture Notes in Control and Information Sciences, Springer Verlag, Berlin 2003.

[11] F. Borrelli, M. Baotić, J. Pekar and G. Stewart, "On the computation of linear model predictive control laws", Automatica, vol.46, pp. 10341041, 2010.

[12] E. A. Wan and R. Van Der Merwe, "The unscented Kalman filter for nonlinear estimation", Adaptive Systems for Signal Processing, Communications, and Control Symposium, AS-SPCC, pp. 153-158, 2000.

[13] V. Lešić, M. Vašak, G. Stojičić, N. Perić, T. M. Wolbank, ”State and Parameter Estimation for Field-oriented Control of Induction Machine Based on Unscented Kalman Filter", International Symposium on Power Electronics, Electrical Drives, Automation and Motion, pp. 409-414, June 2012 